\title{
Making use of geometrical invariants in black hole collisions
}

\author{
John Baker and Manuela Campanelli \\ Albert-Einstein-Institut, Max-Planck-Institut für Gravitationsphysik, Am Mühlenberg 1, D-14476 Golm, Germany
}

(Received 8 March 2000; revised manuscript received 20 July 2000; published 1 November 2000)

\begin{abstract}
We consider curvature invariants in the context of black hole collision simulations. In particular, we propose a simple and elegant combination of the Weyl invariants $I$ and $J$, the speciality index $\mathcal{S}$. In the context of black hole perturbations $\mathcal{S}$ provides a measure of the size of the distortions from an ideal Kerr black hole spacetime. Explicit calculations in well-known examples of axisymmetric black hole collisions demonstrate that this quantity may serve as a useful tool for predicting in which cases the close limit can provide an accurate estimate of the radiation wave form and energy. This makes $\mathcal{S}$ particularly suited to studying the transition from nonlinear to linear dynamics and for an invariant interpretation of numerical results.
\end{abstract}

PACS number(s): 04.25.Dm, 04.25.Nx, 04.30.Db, 04.70.Bw

\section{INTRODUCTION}

Einstein's theory of gravity demands the equivalence of all coordinate representations of gravitational dynamics. This coordinate gauge invariance makes general relativity very simple and beautiful because there are no special families of observers to be considered. On the other hand, though, it can also make it difficult to distinguish whether differences observed in two spacetime representations are true physical (geometrical) differences or gauge differences. A natural way to limit confusion between gauge and physical differences is to work, whenever possible, with geometrically defined scalars, which are invariant under (passive) coordinate transformations. Geometric curvature invariants have a long productive history in the classification and distinction of exact analytic solutions of Einstein's equations, particularly for the algebraic Petrov classification and characterization of curvature singularities [1]. To a lesser extent, curvature invariants have also been applied in the field of numerical relativity, primarily for code testing when evolving exact solutions numerically. Here gauge invariant methods have the distinct advantage that they can be applied to evolutions using numerically generated coordinates which are not understood analytically.

Black hole perturbation theory has recently generated much interest as a model for the late stages of a black hole collision spacetime [2]. When two black holes are close to each other one can simply treat the problem as a single distorted black hole that "rings down" into its final equilibrium state. Perturbative calculations applied in this final regime have become an important tool in the verification and interpretation of numerically generated results [3-5]. More ambitiously, the perturbative approach can be used in conjunction with full scale 3D numerical relativity simulations to directly "take over" and continue a previously computed numerical black hole spacetime [6]. In setting up such a perturbative approach one would like to have a working criteria for when we can expect perturbation theory to be effective based only on numerical data. Some indicators of the potential success of perturbation theory, like for instance the size of the distortions of the apparent horizon, have been previously applied to numerical results [7], but these require es- tablishing a coordinate system, tend to be computationally expensive and none are effective, in particular, for the problem sketched above. In this context it is extremely useful to have a gauge invariant estimate of when perturbation theory can be expected to be effective.

Motivated by this purpose we introduce an invariant quantity $\mathcal{S}$, which geometrically measures local deviation from algebraic speciality, and which we expect to be a very useful tool for numerical and perturbative work involving near-stationary regions of black hole spacetimes. Our invariant index is simple, elegant and can be applied to full 3D numerical evolutions to directly explore the transition from nonlinear to the linear regime [6], or for invariant interpretation of numerical spacetimes. In all well-known examples of axisymmetric black hole collisions we studied, $\mathcal{S}$ has already proven to be very useful.

\section{THE SPECIALITY INDEX}

Curvature invariants are part of the standard analysis of exact solutions of Einstein's equations. From the Weyl tensor, $C_{a b c d}$, which carries information about the gravitational fields in the spacetime, one can algebraically derive two complex curvature invariants usually called $I$ and $J$. These are essentially the square and cube the self-dual part, $\widetilde{C}_{a b c d}$ $=C_{a b c d}+(i / 2) \epsilon_{a b m n} C_{c d}^{m n}$, of the Weyl tensor:

$$
I=\widetilde{C}_{a b c d} \widetilde{C}^{a b c d} \text { and } J=\widetilde{C}_{a b c d} \widetilde{C}^{c d}{ }_{m n} \widetilde{C}^{m n a b} .
$$

These scalars are useful in the algebraic classification of exact solutions. The different algebraic Petrov types are distinguished by the degeneracies among the (up to) four principal null directions (PNDs) associated pointwise with the Weyl tensor. Type $I$ is the algebraically general case with four distinct principal null directions. The other types II, III, D, and $\mathrm{N}$ have at least two coincident PNDs and are referred to as algebraically special. A notable characteristic common to all stationary isolated black hole solutions of general relativity is that they are all algebraically special, of type D, with two pairs of coincident principal null directions (PNDs) at each point. We contend however that for interesting cases involving nontrivial dynamics, perturbed black hole spacetimes are generically not algebraically special, of type I. 
Significantly, the invariants $I$ and $J$ satisfy the relation, $I^{3}=27 J^{2}$ if and only if the Weyl tensor is algebraically special [1]. Since the Weyl tensor in a perturbed black hole spacetime is not expected to be algebraically special, we expect $I^{3} \neq 27 J^{2}$. Our proposal, then, is to use this relation to construct an invariant index for algebraic speciality as a local measure of the size of the distortions from some background black hole. This violation can be in general quantified by considering the following speciality index:

$$
\mathcal{S}=\frac{27 J^{2}}{I^{3}} .
$$

Note that, unlike $I$ and $J$, the peeling theorem implies no characteristic falloff for $\mathcal{S}$. For the unperturbed algebraically special background Kerr spacetime $\mathcal{S}=1$. In the perturbed spacetime we generically expect $\mathcal{S}=1+\Delta \mathcal{S}$, and the size of the deviation $\Delta \mathcal{S} \neq 0$ can be used as a guide to predicting the effectiveness of black hole perturbation theory.

The theory of perturbations on a background Kerr spacetime was worked out first by Teukolsky [8] and has been extensively studied by many authors [9-12]. In this context it is natural to use the Newman-Penrose decomposition of the Weyl tensor into five complex quantities, $\psi_{0}, \psi_{1}, \psi_{2}$, $\psi_{3}$, and $\psi_{4}$, defined with respect to some choice of a null tetrad basis. In terms of the Weyl components, for an arbitrary tetrad choice:

$$
\begin{aligned}
& I=3 \psi_{2}^{2}-4 \psi_{1} \psi_{3}+\psi_{4} \psi_{0}, \\
& J=-\psi_{2}^{3}+\psi_{0} \psi_{4} \psi_{2}+2 \psi_{1} \psi_{3} \psi_{2}-\psi_{4} \psi_{1}^{2}-\psi_{0} \psi_{3}^{2} .
\end{aligned}
$$

For any type D spacetime such as Kerr, a tetrad can be conveniently chosen such that only $\psi_{2}$ is non-vanishing. By expressing $\mathcal{S}$ with respect to any perturbation of such a tetrad we find that

$$
\mathcal{S}=1-3 \epsilon^{2} \frac{\psi_{0}^{(1)} \psi_{4}^{(1)}}{\left(\psi_{2}^{(0)}\right)^{2}}+\mathcal{O}\left(\epsilon^{3}\right)
$$

were $\epsilon$ is a perturbation parameter, and the superscript (0) and (1) stand respectively for background and first order pieces of the perturbed Weyl scalars. Thus, the lowest order term in the deviation is second order in the perturbation parameter $\epsilon$. In the perturbative context this means that, when the speciality index is significantly different from unity, one can see that a potentially second order quantity has become significant, and the first order theory should no longer be trusted. For the case of Schwarzschild Eq. (4) can be reexpressed in terms of the gauge invariant Moncrief functions [10].

\section{EXAMPLE APPLICATIONS}

For considering deviations of $\mathrm{S}$ from unity it is natural to think of deviations by some factor with the strongest at $\mathcal{S}$ $=0$ and $\mathcal{S}=\infty$ when $J$ or $I$ vanish. In order to make the symmetry between $\mathcal{S}$-too large and $\mathcal{S}$-too small deviations more apparent in graphical representation it is useful to con- sider a rescaled form $\mathcal{S}_{\text {norm }}=1+(\mathcal{S}-1) / \sqrt{|\mathcal{S}|^{2}+1}$, which is bounded in magnitude between 0 and 2 . A deviation of a "factor of two" for $\mathcal{S}$ corresponds to an additive deviation of the approximately \pm 0.5 in $\mathcal{S}_{\text {norm }}$. We take this as a reasonable cutoff, to be checked a posteriori, for values of $\mathcal{S}_{\text {norm }}$ above which we cannot expect perturbation theory to be applicable. In regions where this condition is violated we can expect significant violations of the perturbative dynamics. To test our interpretation of $\mathcal{S}$ we have considered some wellstudied cases of axisymmetric black hole collisions. We find it useful to consider the location of such violating regions with respect to the background black hole horizon and the perturbative " potential barrier." As is well known, perturbative black hole dynamics are governed by a wave equation with a potential concentrated in the vicinity of $r=2 M$ in the isotropic coordinates used for our examples. The potential has the effect of largely preventing waves from crossing this region.

The examples we consider here all correspond to evenparity modes implying that $\mathcal{S}$ is real, so we leave out reference to its imaginary component in the following discussion. We first consider the case of two initially resting equal-mass black holes. This initial configuration is represented by the equal-mass time-symmetric Misner datasets parametrized by $\mu_{0}$ as a measure of the initial separation. In this case, Schwarzschild black hole perturbation theory has been shown to provide a very good estimate of the total radiated energy for cases with $\mu_{0}<1.8$ even though the black holes share a common apparent horizon only when $\mu_{0}<1.36$. Comparisons with numerical calculations [4] and second order calculations [13] have demonstrated that the linear perturbation approach overestimates the radiation energy by only a factor of two up to $\mu_{0}=1.8$ but beyond that the differences grow quickly. Second order perturbations have been applied in this case as a useful tool for assessing the domain of validity of perturbation theory. We can obtain similar conclusions by applying our speciality index test. Figure 1 shows the initial values of $\mathcal{S}_{\text {norm }}$ along the equator for Misner data, at several initial separations. In isotropic coordinates, as used in previous studies, the horizon of the background black hole is located at $r=0.5 \mathrm{M}$ and location of the perturbative "potential barrier" is near $r=2 M$. First consider the case, $\mu_{0}=1.2$. While there is small region near the horizon where the criterion fails, this region is well inside the potential barrier which should prevent any local errors in the perturbative dynamics from having a significant effect on the outgoing radiation. In the marginal $\mu_{0}=1.8$ case, the error in $\mathcal{S}_{\text {norm }}$ begins to be significant near the potential barrier, and the radiation should be somewhat affected. For larger values of $\mu_{0}$ the violation is significant in the vicinity of the potential barrier itself, invalidating the perturbation dynamics. The sudden drop in the value of $\mathcal{S}_{\text {norm }}$ in these cases makes our interpretations insensitive to the choice of a cutoff.

Another well-studied case is the collision of black holes with non-vanishing initial linear momentum $P$. Here again, first order perturbation theory has been very successful, unexpectedly providing a good estimate of the radiation energies even for large values of $P$ [14]. We consider configura- 


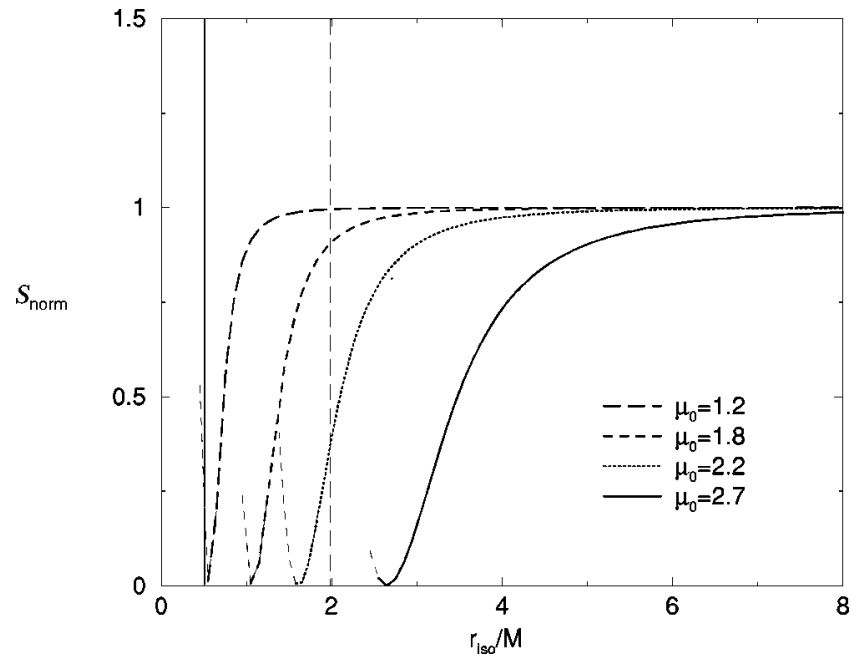

FIG. 1. The specialty index for Misner data. The equatorial values of $\mathcal{S}_{\text {norm }}$ shown in this plot allow the interpretation that perturbation theory can work for cases of $\mu_{0}<1.8$ but not higher. The "potential barrier" near $r_{i s o}=2 M$ prevents the violation of perturbative dynamics inside near the horizon at $r=0.5 \mathrm{M}$, from affecting the radiation. For larger separations the interpretation of a potential barrier at $r_{\text {iso }}=2 M$ is itself invalidated.

tions corresponding to a fixed $\mu=1.5$, for various values of $P$. The corresponding initial values of $\mathcal{S}_{\text {norm }}$ are shown in Fig. 2. The presence of momentum in the initial slice introduces qualitatively different features to the initial values of $\mathcal{S}$ exhibiting now a region of $\mathcal{S}>1$ which falls off more slowly at large $r$. There is no question in this case of the location of this region, but rather the magnitude of the violation. In this case the violation seems to grow quadratically with $P$ and reaches our cutoff just after $P=M$. Suggesting perturbation theory should be successful up to this $P \sim M$. The prediction

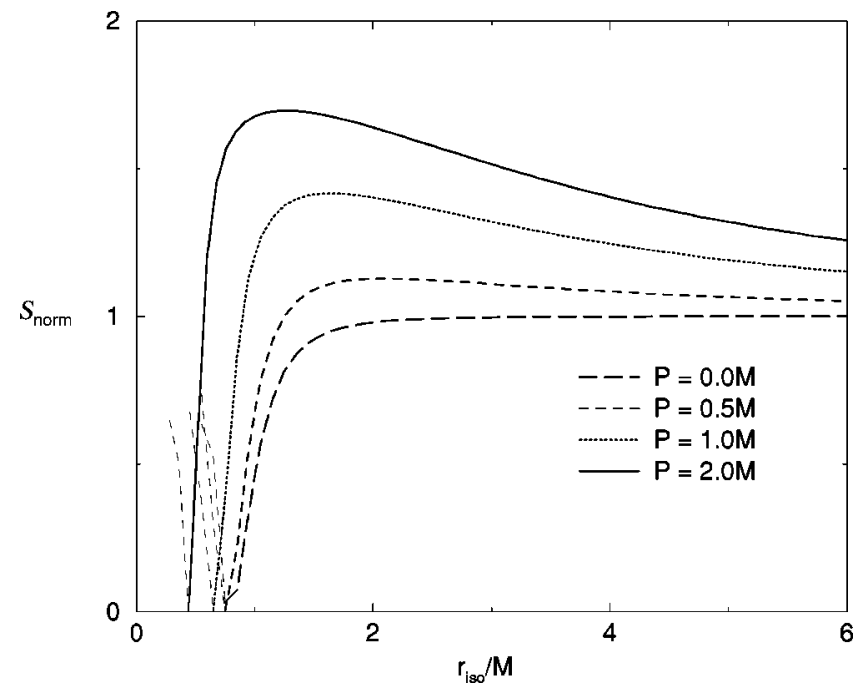

FIG. 2. Boosted black holes. The initial inward linear momentum of the black holes results in qualitatively different features in the equatorial $\mathcal{S}_{\text {norm }}$ values. These curves correspond to separations of $\mu_{0}=1.5$ and suggest that perturbation theory should be good below $P=M$.
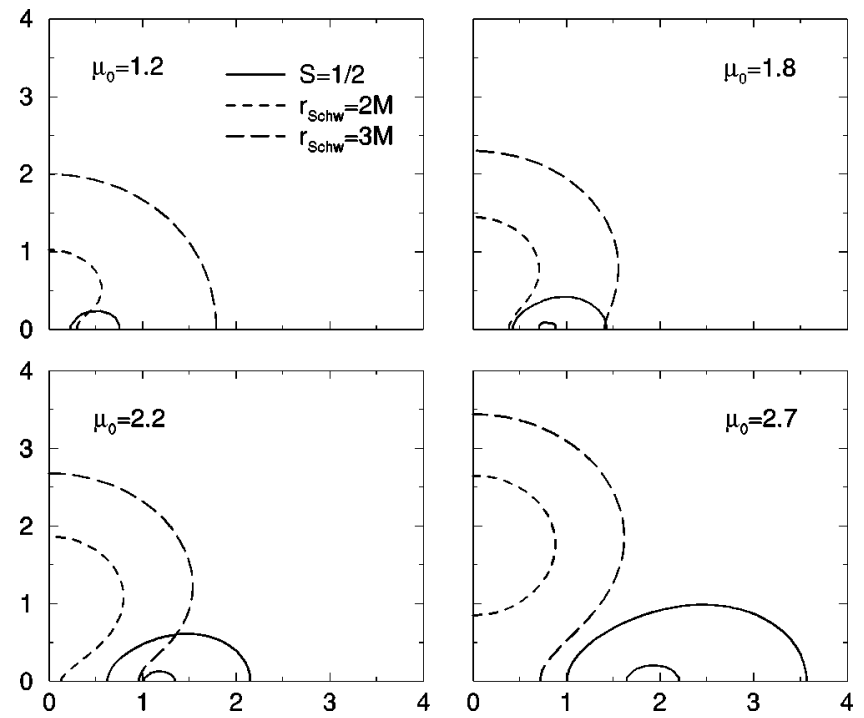

FIG. 3. Fully invariant analysis. Here we reexamine the Misner results, for the $\mu_{0}=1.2,1.4,1.8,2.2$ cases respectively, showing the full $2 \mathrm{D}$ picture in a quadrant of the $x z$ plane. The results of the analysis this time provided in terms of an invariantly defined background and $r_{S c h w}=2 M$ horizon and potential barrier near $r_{S c h w}$ $=3 M$. The solid curve indicates the $\mathcal{S}=1 / 2$ surface.

of our prescription is consistent with the accuracy to $10 \%$ for radiation energies shown in Ref. [14] for $P<M$ but does not explain the mere doubling of this discrepancy out to $P$ $=3 \mathrm{M}$.

In the above analysis we have considered the location of the $\mathcal{S} \neq 1$ regions in relation to the local properties of the "background" black hole. The specification of the background metric in this standard treatment is not itself gauge independent though. We can evaluate the applicability of a perturbative treatment in a gauge invariant way by utilizing a gauge-invariant specification of the background. A simple way to do this is to specify the background Schwarzschild radial coordinate by $r_{S c h w}^{6}=3 M^{2} / I$. The location of the horizon and potential barrier are then found with respect to this coordinate. A two-dimensional representation of the results for Misner initial data is given in Fig. 3. These plots show three curves, representing the locations of the background horizon, the potential barrier, and the $\mathcal{S}=0.5$ surfaces in a quadrant of the $x z$-plane. The qualitative features observed in the preceeding interpretation of $\mathcal{S}$ for the Misner problem, are reproduced precisely in this more complete, fully invariant perspective.

\section{DISCUSSION}

We have identified a gauge invariant quantity which provides a particularly interesting local reduction of the geometric data in a (numerical) black hole spacetime. We foresee three areas of application where $\mathcal{S}$ should be a useful quantity: perturbation studies, which we have discussed in detail, numerical spacetime interpretation, and numerical code testing. In the context of perturbation theory we have demonstrated that $\mathcal{S}$ provides an invariant criterion for predicting when perturbation theory might provide a reliable approxi- 
mation for part of a black hole spacetime. This method has the advantage over other estimates such as apparent horizon formation that it is genuinely gauge invariant. In practice it can serve as simple alternative to second order perturbation theory, but our prediction does not provide such a direct validation of a perturbative calculation. Knowing that $\mathcal{S} \sim 1$ does not, for example, identify the appropriate background spacetime, a vital step in any application, but it is very useful predictor of when perturbation theory may be a useful alternative to numerical simulation in a generic black hole spacetime. The quantity $\mathcal{S}$ itself is not restricted to perturbation studies but should also be useful in the general interpretation of numerical spacetimes. $\mathcal{S}$ is uniquely valuable in this context because it is a gauge invariant quantity, responsive to dynamical disturbances, such as radiation, but with no characteristic (peeling) falloff behavior. Thus $\mathcal{S}$ can indicate typical dynamical disturbances directly, without reference to any additional structure. As an example, looking at $\mathcal{S}$ in numerical simulations, Misner data evolve after a short time to qualitatively resemble the black hole data with inward momentum discussed above. After longer evolutions in this family $\mathcal{S}$ tends to approach unity in the horizon-potential region with evidence of radiation moving away in both di- rections. In this context we also note that the presence of an isolated horizon [15], a recent construct of growing theoretical interest in black hole spacetimes, implies locally that $\mathcal{S}$ $=1$. Lastly, because $\mathcal{S}$ is an invariant with often predictable behavior, it can be very useful in numerical code testing. In Kerr spacetimes, for example, $\mathcal{S}=1$ exactly in any coordinates. Also in typical cases of Bowen-York binary black hole data that we have looked at $\mathcal{S}$ falls off quickly toward unity away from the black holes. In light of its simplicity and straightforward significance, we expect $\mathcal{S}$ to become a standard, very useful tool for analyzing numerically generated spacetimes and interpreting their physical content.

\section{ACKNOWLEDGMENTS}

We thank Miguel Alcubierre, Carlos Lousto, Richard Price and Jorge Pullin for their support and helpful suggestions. This work was supported by AEI. M. C. was supported through a Marie-Curie Foundation grant HPMF-CT-199900334. All our numerical computations have been performed with a full 3D code, Cactus, on an 8 GB SGI Origin 2000 with 32 processors at AEI.
[1] M. M. D. Kramer, H. Stephani, and E. Herlt, Exact Solutions of Einstein's Field Equations (Cambridge University Press, Cambridge, England, 1980).

[2] R. H. Price and J. Pullin, Phys. Rev. Lett. 72, 3297 (1994).

[3] P. Anninos, R. H. Price, J. Pullin, E. Seidel, and W.-M. Suen, Phys. Rev. D 52, 4462 (1995).

[4] J. Baker, A. Abrahams, P. Anninos, S. Brandt, R. Price, J. Pullin, and E. Seidel, Phys. Rev. D 55, 829 (1997).

[5] J. Baker, S. R. Brandt, M. Campanelli, C. O. Lousto, E. Seidel, and R. Takahashi, Phys. Rev. D. (to be published), gr-qc/9911017.

[6] J. Baker, B. Brügmann, M. Campanelli, and C. O. Lousto, Class. Quantum Grav. 17, L149 (2000).

[7] A. M. Abrahams, S. L. Shapiro, and S. A. Teukolsky, Phys. Rev. D 51, 4295 (1995).
[8] S. A. Teukolsky, Astrophys. J. 185, 635 (1973).

[9] M. Campanelli and C. O. Lousto, Phys. Rev. D 59, 124022 (1999).

[10] M. Campanelli and C. O. Lousto, Phys. Rev. D 58, 024015 (1998).

[11] M. Campanelli, W. Krivan, and C. O. Lousto, Phys. Rev. D 58, 024016 (1998).

[12] M. Campanelli, C. O. Lousto, J. Baker, G. Khanna, and J. Pullin, Phys. Rev. D 58, 084019 (1998).

[13] R. J. Gleiser, C. O. Nicasio, R. H. Price, and J. Pullin, Phys. Rev. Lett. 77, 4483 (1996).

[14] C. O. Nicasio, R. J. Gleiser, R. H. Price, and J. Pullin, Phys. Rev. D 59, 044024 (1999).

[15] A. Ashtekar, C. Beetle, and S. Fairhurst, Class. Quantum Grav. 17, 253 (1999). 